\title{
Visualisation hydrodynamique de l'écoulement dans une maquette de turbomachine axiale
}

\author{
Hydrodynamic visualization of flow using a scale-model \\ of an axial flow turbomachine
}

\author{
H. Werlé
}

ONERA, Chatillon

Grâce à des visualisations effectuées dans une cuve hydraulique verticale de l'ONERA, il a été possible d'entreprendre l'étude physique de l'écoulement instationnaire dans une maquette limitée à une route mobile isolée ou en présence d'un redresseur.

De ces résultats obtenus à faible vitesse (1), se dégage un certain nombre de schémas qui correspondent au cas d'un fonctionnement au régime d'adaptation ou à débit partiel (décollement tournant). La présente synthèse analyse les phénomènes de couche limite et de décollement observés, ainsi que leur évolution en fonction du pas relatif et du jeu d'extrémité des aubes mobiles.

\section{Introduction}

Les visualisations effectuées à l'intérieur d'une turbomachine et analysées dans cet article ont pour but d'accéder à une meilleure connaissance de phénomènes particulièrement complexes puisqu'il s'agit d'un écoulement tridimensionnel, instationnaire et généralement turbulent. Ces phénomènes sont bien souvent difficiles à observer en raison des reflets et ombres portées qui se multiplient autour de la carène transparente d'une telle maquette comportant des éléments mobiles et fixes.

Cette recherche s'inscrit dans le cadre de l'étude fondamentale de l'écoulement dans les turbomachines, entreprise à l'ONERA aussi bien par la Direction Energétique [1-2] que par la Direction Aérodynamique [3]. En ce qui concerne les visualisations faites à la cuve, une première publication [4] avait donné les résultats obtenus avec une roue mobile équipée de 20 aubes cylindriques (corde $l=25 \mathrm{~mm}$ ), en les

(1) Quelques exemples ont été groupés dans le film ONERA 707 présenté en cours de séance. confrontant au cas d'une grille d'aubes plane mobile; une deuxième publication [5] avait analysé l'écoulement autour d'une roue mobile avec 20 aubes de corde plus grande (corde $l=50 \mathrm{~mm}$ ), observées non seulement suivant des axes fixes, mais aussi suivant des axes liés à la roue grâce à un montage optique utilisant un rotoscope [6].

Le présent article commence par effectuer une synthèse de ces résultats antérieurs, puis prolonge cette étude en examinant le cas d'une grille d'aubes mobile sans jeu d'extrémité (carène toumante) résumant ainsi l'essentiel du film ONERA 707 [10] présenté au cours de la session.

\section{Technique expérimentale}

De toutes les caractéristiques dimensionnelles de la cuve [4] et de la maquette [5] ne seront rappelées ici que les plus importantes :

- l'installation utilisée (Fig. 1) est verticale et fonctionne simplement par vidange sous l'effet de la gravité. La vitesse débitante $V_{1}$ en amont de la roue dans la veine annulaire (rayon du carter $R=146 \mathrm{~mm}$, rayon du moyeu $=100 \mathrm{~mm}$ ) est contrôlée par un ensemble vanne-débitmètre. La vitesse de rotation $(N)$ de la roue est imposée et réglable, d'où l'angle relatif $\beta_{1}$ donné $\operatorname{par} \frac{V_{1}}{2 \pi r N}=\operatorname{cotg} \beta_{1}$. Etant donné le calage $\gamma=45^{\circ}$ et la cambrure $\varphi=32^{\circ} 30$ des 20 aubes cylindriques, l'attaque idéale se trouve réalisée pour $\beta_{1}=61^{\circ} 15$. Notons enfin les caractéristiques des aubes utilisées : - pour $l=25 \mathrm{~mm}$, pas relatif $s / l=1,56$ à $0,85 R$ ( $\simeq$ rayon moyen) et jeu d'extrémité $0,7 \% R$, - pour $l=50 \mathrm{~mm}, s / l=0,78$ à $0,85 R$ et jeu d'extrémité $1 \% R$. 


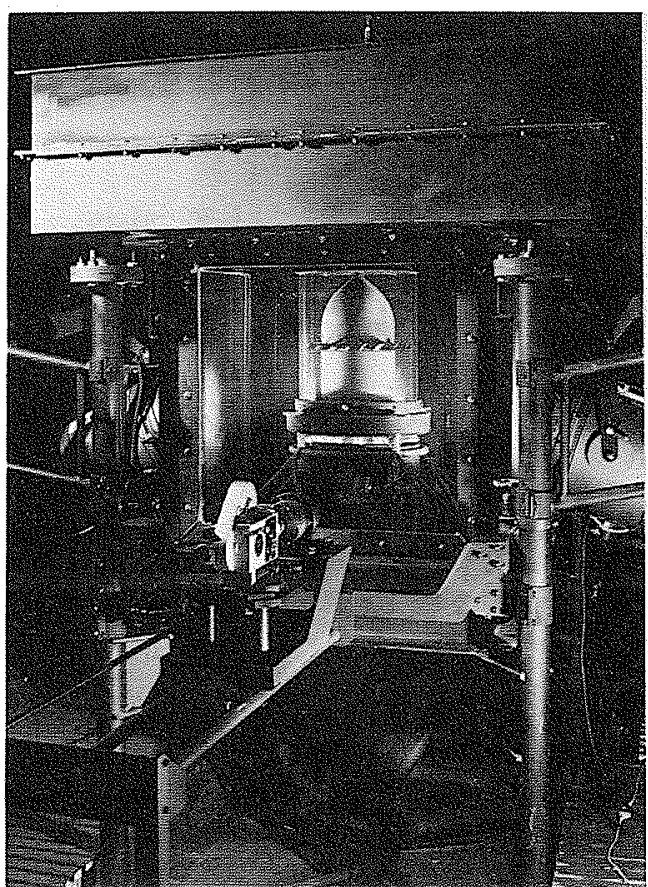

Figure 1 - Vue de la cuve hydraulique avec sa maquette de turbomachine axiale.

Nota : on distingue au premier plan l'ensemble camérarotoscope servant aux prises de vues suivant des axes liés à la roue mobile et mettant en jeu des miroirs disposés sous le modèle.

Le montage "turbomachine" a été conçu de manière à permettre l'emploi des techniques de visualisation habituellement utilisées dans les autres tunnels hydrodynamiques de l'ONERA [7] :

- l'écoulement pariétal est mis en évidence par des émissions colorées d'une densité égale à un et issues d'éléments fixes ou mobiles (phénomènes de couche limite sur les aubes, sur la carène ou le moyeu, décollements, etc.) : voir par exemple figure $3 b, c$ et $g$;

- l'allure et le caractère stationnaire ou non de l'écoulement extérieur sont révélés par les filets colorés émis à partir d'une rampe fixe disposée à l'amont des aubes, par exemple à la mi-envergure (voir fig. $3 d$, $e$ et $g$ ) ou au voisinage de la carène ( $F$ ig. $5 d$ et $e$ ). Dans ce cas, des prises de vues cinématographiques permettent d'enregistrer l'évolution des phénomènes en fonction du temps (Fig. $3 d$ et $e$ );

- la méthode des bulles d'air fournit des images de l'écoulement en amont des aubes, entre les aubes ou en aval, suivant des plans de coupes longitudinaux (voir Fig. 2). Alors qu'un temps d'exposition court (obtenu par ex. à l'aide d'un flash) suffit, dans le cas des émissions de colorant, pour obtenir directement des filets colorés, il est au contraire indispensable, dans le cas des bulles d'air, de conserver un temps de pose suffisant pour leur laisser tracer des éléments de trajectoire représentant les lignes de courant des parties stationnaires ou seulement l'allure moyenne des parties instationnaires (voir par ex. Fig. $3 a, f$ et $h$ et Fig. $5 a, b$ et $c$ ).

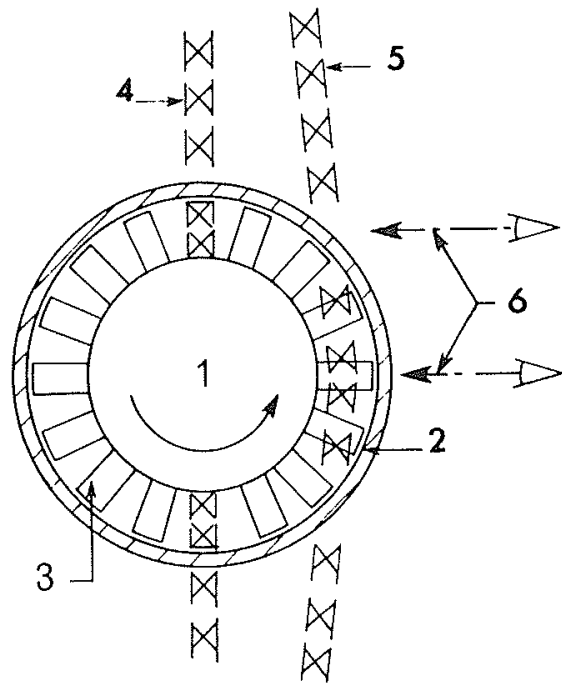

Figure 2 - Schéma de prise de vues (coupe transversale à la hauteur de la roue mobile).

1 : moyeu ;

2 : carène (carter)

3 : roue mobile ( 20 aubes)

4: tranche éclairée disposée suivant l'axe du modèle ;

5 : tranche éclairée disposée suivant la mi-envergure des aubes;

6: axes de visée correspondants (tranches longitudinales).

Les bulles d'air ont aussi servi à préciser l'allure de l'écoulement dans des plans de coupe transversaux observés, à l'aide de miroirs disposés en aval, suivant des axes fixes ou mobiles (rotoscope). Le lecteur intéressé trouvera dans $[5,10]$ la description de ce montage et toutes les indications utiles concernant cette méthode plus délicate à mettre en œuvre.

\section{Essais au régime de fonctionnement adapté}

Le fonctionnement au régime d'adaptation (Fig. 3) a été obtenu pour un rapport $\frac{V_{1}}{2 \pi r N}$ égal à 0,55 à l'extrémité des aubes cylindriques non vrillées, c'està-dire à l'attaque idéale. Dans ces conditions, l'écoulement amont conserve jusqu'au voisinage immédiat de la roue son caractère stationnaire et laminaire, uniforme en dehors des couches limites : lignes de courant parallèles et confondues avec les lignes d'émission (colorant) et trajectoires de bulles d'air, et ceci reste vrai le long du moyeu et de la carène.

L'écoulement moyen (visualisé par bulles d'air sur les clichés à temps d'exposition assez long) en aval de la roue présente un caractère nettement plus turbulent et à peu près uniformément défléchi dans le sens de la rotation (Fig. $3 a$ ). Un temps d'exposition plus court permet de distinguer la trace des sillages minces qui prolongent les aubes à corde plus grande et d'un pas relatif réduit dans le cas de la deuxième roue (Fig. $3 f$ ).

Bien entendu, ce sillage qui se déplace avec les aubes dont il est issu, perturbe à chaque passage l'écoulement turbulent autour des aubes fixes d'un redresseur dis- 

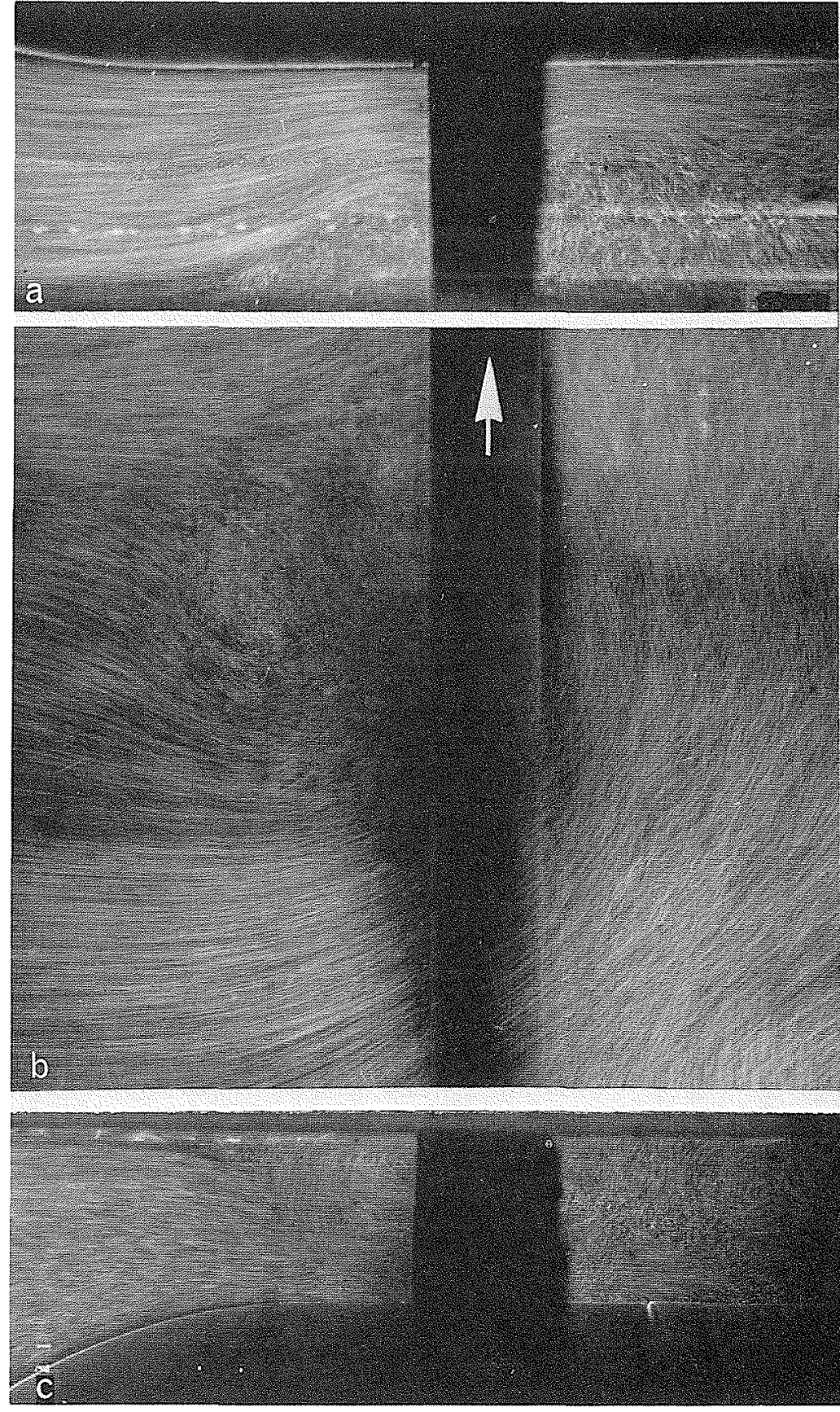
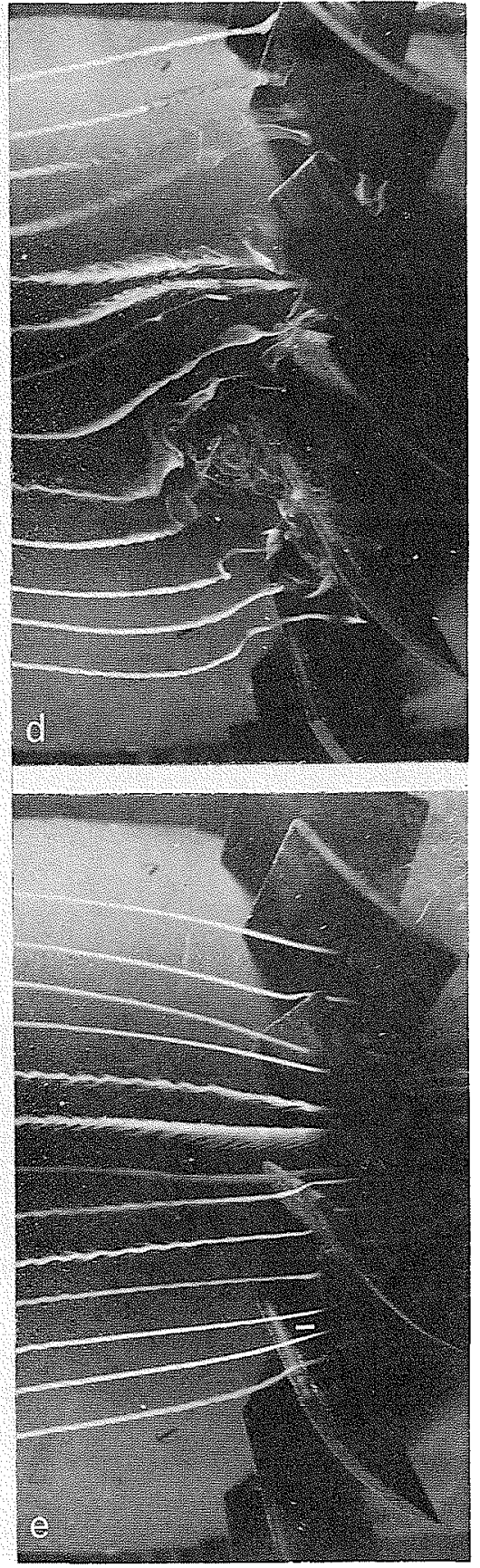

Figure 3 - Ecoulement dans une maquette de turbomachine axiale : essai au régime de fonctionnement correct. a b c de : roue isolée $\left(l=25 \mathrm{~mm}, N=0,4 \mathrm{tr} / \mathrm{s}, \mathcal{R}_{e_{l}} \simeq 10^{4}\right)$

$f \quad$ : roue isolée $\left(l=50, \mathrm{~mm}, N=0,1 \mathrm{tr} / \mathrm{s}, \mathcal{R}_{e_{l}} \simeq 1 / 2.10^{4}\right)$;

$g h \quad:$ essai avec Iedresseur à 8 aubes

a fh : tranches suivant la mi-envergure deș aubes (visualisation par bulles d'air);

deg : émissions à partir d'une rampe fixe

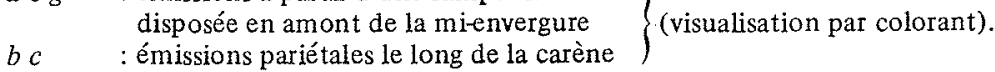

posé derrière la roue mobile (Fig. $3 h$ ) : on distingue notamment le déplacement momentané du point d'arrêt au bord d'attaque où se forme un décollement localisé périodique.

Les visualisations à l'aide de colorants recoupent et complètent les résultats précédents : les aubes mobiles coupent périodiquement les filets colorés parallèles émis au sein du fluide par une rampe amont; côté aval, ces filets cessent de représenter des lignes de courant, mais révèlent la structure à tourbillons alternés du sillage des aubes (Fig. $3 d e$ ). Le long du carter, les émissions pariétales montrent le "fraisage" de la couche limite produit par les aubes mobiles, dont l'effet d'obstacle subsiste en dépit du jeu d'extrémité et se traduit par un décollement localisé sur la carène des deux côtés de chaque aube (Fig. $3 b c$ ). Enfin, 
lors des essais effectués en présence d'un redresseur (Fig. $3 g$ ), le colorant émis au bord d'attaque d'une de ses aubes fixes confirme le caractère tourbillonnaire du décollement localisé qui s'y forme et s'échappe vers l'aval, comme sur un profil oscillant.

De l'ensemble de ces essais peut être dégagé le schéma donné par la figure 4 . En se repérant suivant des axes fixes, on enregistre au sein du fluide et le long des parois les oscillations intrinsèques qui résultent du passage de chaque pale et qui assurent la transition entre l'écoulement stationnaire amont et l'écoulement instationnaire aval.

Observé suivant des axes liés à la roue, l'écoulement retrouve son caractère stationnaire; en effet, mis à part les altemances du sillage émis par les aubes, les

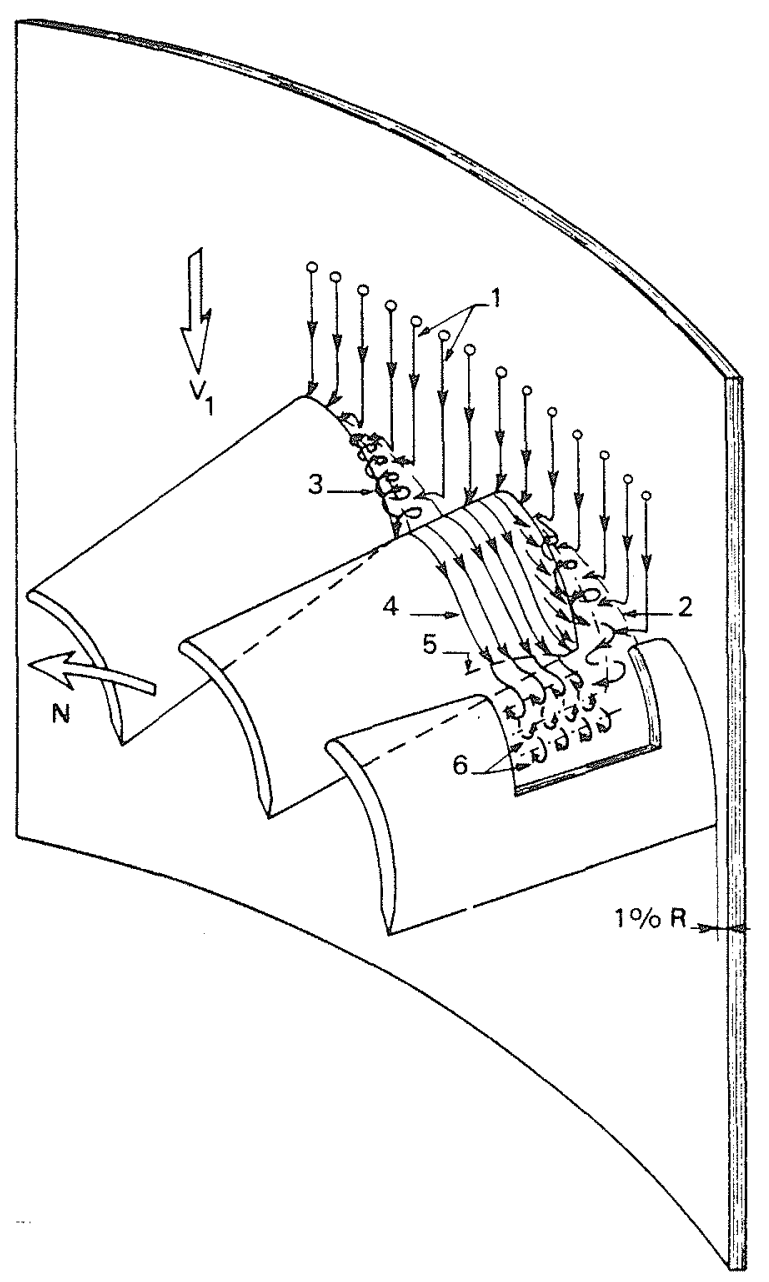

Figure 4 - Schéma de l'écoulement dans une maquette de turbomachine axiale

(Régime de fonctionnement correct :

$$
\left.\mathcal{R}_{e_{l}} \approx 10^{4} \text { et } \frac{V_{1}}{2 \pi r N}=0,55 \text { pour } \frac{r}{R}=0,99\right)
$$

1 : lignes de courant pariétales le long de la carène ;

2 : décollement devant le flux d'intrados contournant l'extrémité des aubes;

3 : tourbillon marginal ;

4: lignes de courant pariétales sur l'extrados convexe des aubes;

5 : décollement près du bord de fuite des aubes;

$6:$ tourbillons alternés caractérisant le sillage des aubes. petits décollements sur ces aubes et sur la carène restent de volume constant et d'importance égale sur toutes les aubes mobiles (essai sans redresseur).

\section{Fonctionnement à débit partiel}

Le régime hors adaptation qui apparaît pour un rapport inférieur à celui qui correspond au régime correct, soit par exemple pour $\frac{V_{1}}{2 \pi r N}=0,275$ en tête des aubes, est caractérisé par la formation d'un décollement tournant qui s'étend en amont de la roue mobile et sur plusieurs aubes. Cette zone décollée qui se présente sous la forme d'une ou plusieurs poches distinctes, se déplace dans le même sens, mais à une vitesse inférieure à celle des aubes. Le volume moyen de chacune de ces poches de change pas à $\frac{V_{1}}{2 \pi r N}$ constante mais peut varier d'une poche à l'autre. Dans une tranche marginale (Fig. $5 b$ ), le passage d'une telle zone décollée est marqué par une variation notable de l'orientation des trajectoires des bulles d'air à l'entrée et à la sortie des canaux interaubes : elle traduit les différentes phases du phénomène : débit, blocage et même inversion de courant dans ces canaux.

Dans une tranche diamétrale (Fig. $5 a c$ ), les bulles d'air confirment que le décollement tournant se produit toujours du côté de la carène, que la progression vers l'amont du décollement (trace de sa frontière dans le plan visualisé) est plus lente que son recul lorsque se rétablit le courant entre les aubes, enfin que la poche décollée se développe et s'étend vers l'amont quand l'angle d'attaque $\beta_{1}$ augmente à $N$ constante. En comparant les coupes $5 a$ et $5 c$, on constate aussi que la structure de ce décollement ne change guère sous l'effet d'une nette variation du pas relatif, d'ailleurs liée à une légère modification du jeu d'extrémité et du nombre de Reynolds dans ces essais.

Les visualisations par colorant recoupent tous ces résultats : par exemple, désorganisation des filets au passage d'une poche décollée (Fig. $5 d$ ) et au contraire, convergence des filets qui s'engouffrent dans le canal situé entre deux poches successives (Fig. $5 e$ ). Les films de visualisation pris au cours des essais révèlent enfin que le phénomène de décollement est lent à s'établir où à se modifier en fonction du coefficient de débit et qu'il reste soumis à un effet d'hystérésis. Le schéma de la figure 6 tente une synthèse de toutes ces observations qui précise la structure de la poche de recirculation issue d'un point de partage amont et résultant de l'extension, puis de la jonction des différents décollements qui affectent l'extrémité des aubes concernées.

\section{Influence du jeu d'extrémité}

Ce schéma semble mettre en lumière le rôle important du jeu existant à l'extrémité libre des aubes. L'influence de ce paramètre avait d'ailleurs fait l'objet d'une étude antérieure au tunnel hydrodynamique 

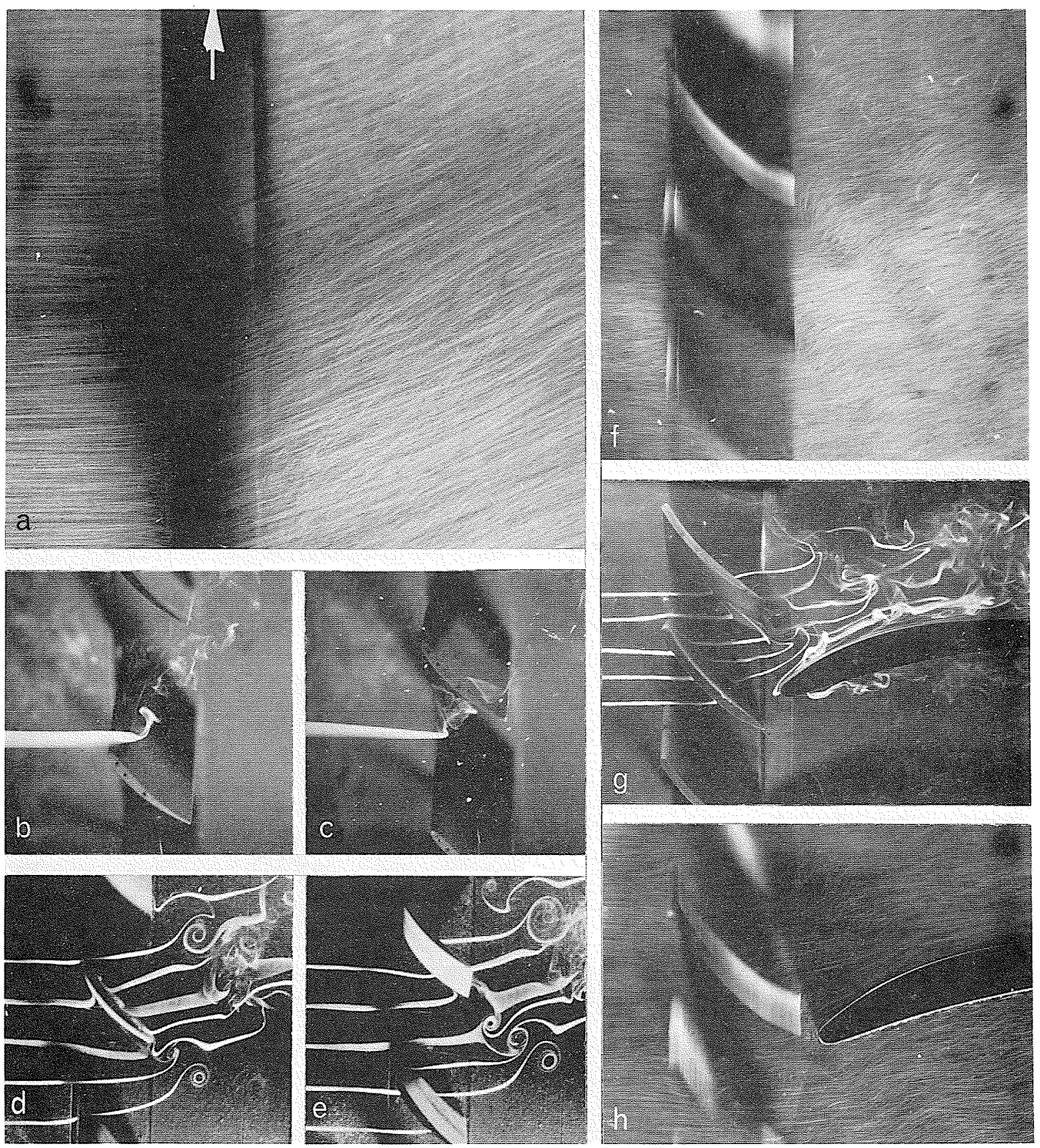

Figure 5 - Ecoulement dans une maquette de turbomachine axiale : fonctionnement hors adaptation avec décollement tournant.

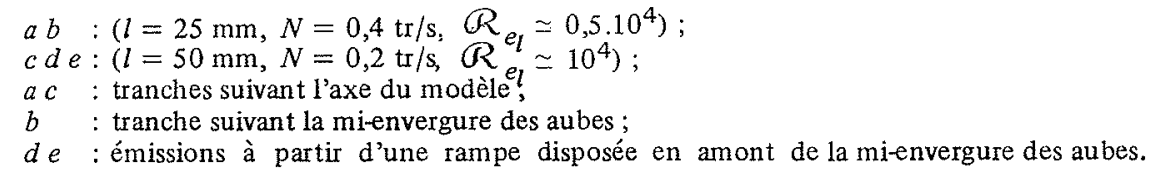

en régime stationnaire [8]. C'est pourquoi une dernière campagne d'essai a été effectuée avec une roue à aubes sans jeu, parce que munie d'une partie de carène tournante (Fig. 7a), bien que cette solution soit difficilement adaptable aux turbomachines aéronautiques.

Pour un régime de fonctionnement correct (Fig. $7 b c)$, il est indéniable que la rotation de la carène (comme celle du moyeu) et l'entraînement de la couche limite qui en résulte [9] assurent localement des conditions plus proches de l'attaque idéale et évitent bien sûr le "fraisage" de la couche limite qui se produit le long d'une paroi fixe.

Par contre, dans le cas d'un fonctionnement hors adaptation (Fig. $7 d e f$ ) ce procédé tel qu'il a été essayé, n'a pas permis d'éviter la formation ou de limiter l'importance du décollement tournant. 


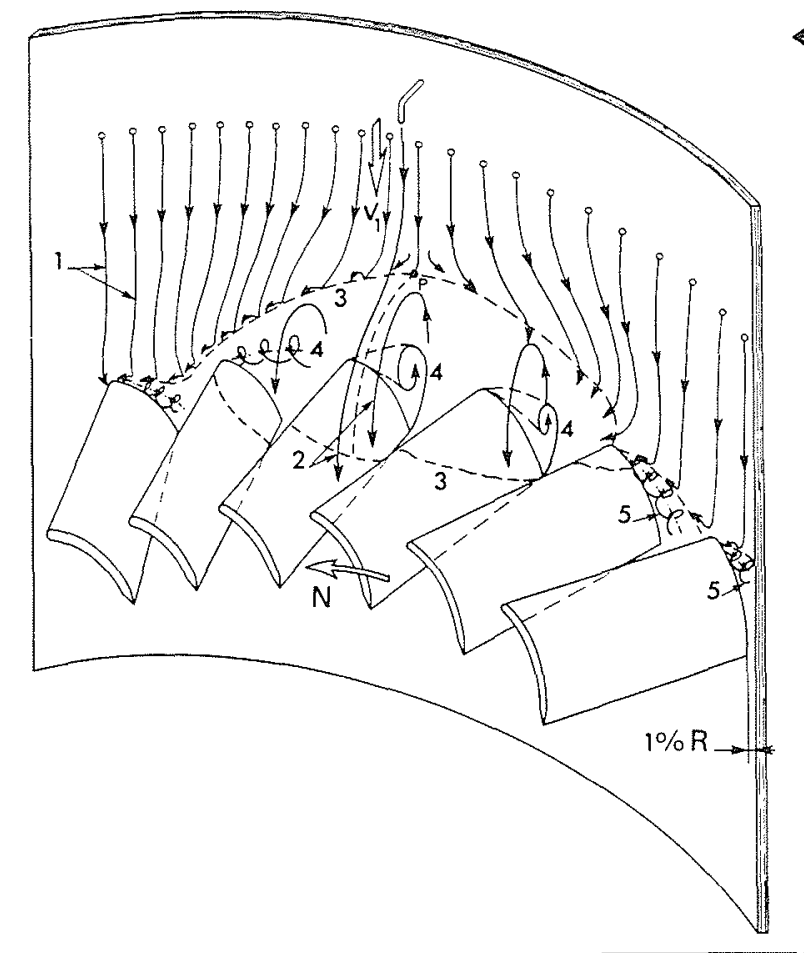

$\leftarrow$ Figure 6 - Schéma de l'écoulement dans une maquette de turbomachine axiale. (fonctionnement hors adaptation avec

$N=0,2 \mathrm{tr} / \mathrm{s} ;-\mathcal{R}_{e_{l}} \sim 0,59 \cdot 10^{4} ;-\frac{V_{1}}{2 \pi r N}=0,275$ pour $\left.\frac{r}{R}=0,99\right)$.

1 : lignes de courant pariétales le long de la carène ;

2 : lignes de courant non pariétales à l'extérieur ou à l'intérieur de la poche décollée ;

3 : trace sur la carène et les aubes de la frontière de la poche décollée;

4: zone tourbillonnaire à l'extrémité des aubes concernées par la poche éclatée (tourbillon marginal éclaté);

5 : tourbillon marginal des aubes situées en dehors du décollement tournant;

$P$ : point de partage au sommet de la poche décollée.

Figure 7 - Ecoulement dans une maquette de turbomachine axiale : essais d'une roue à aubes sans jeu d'extrémité (carène tournante).

$a$ : schéma (coupe).

1: moyeu;

2: roue mobile;

3: partie tournante de la carène ;

4: carène fixe ;

$b c$ : essai au régime de fonctionnement correct;

$d$ e $f$ : fonctionnement hors adaptation avec décollement tournant ; $b d e$ : tranches suivant l'axe du modèle ;

c $f$ : tranches suivant la mienvergure des aubes.
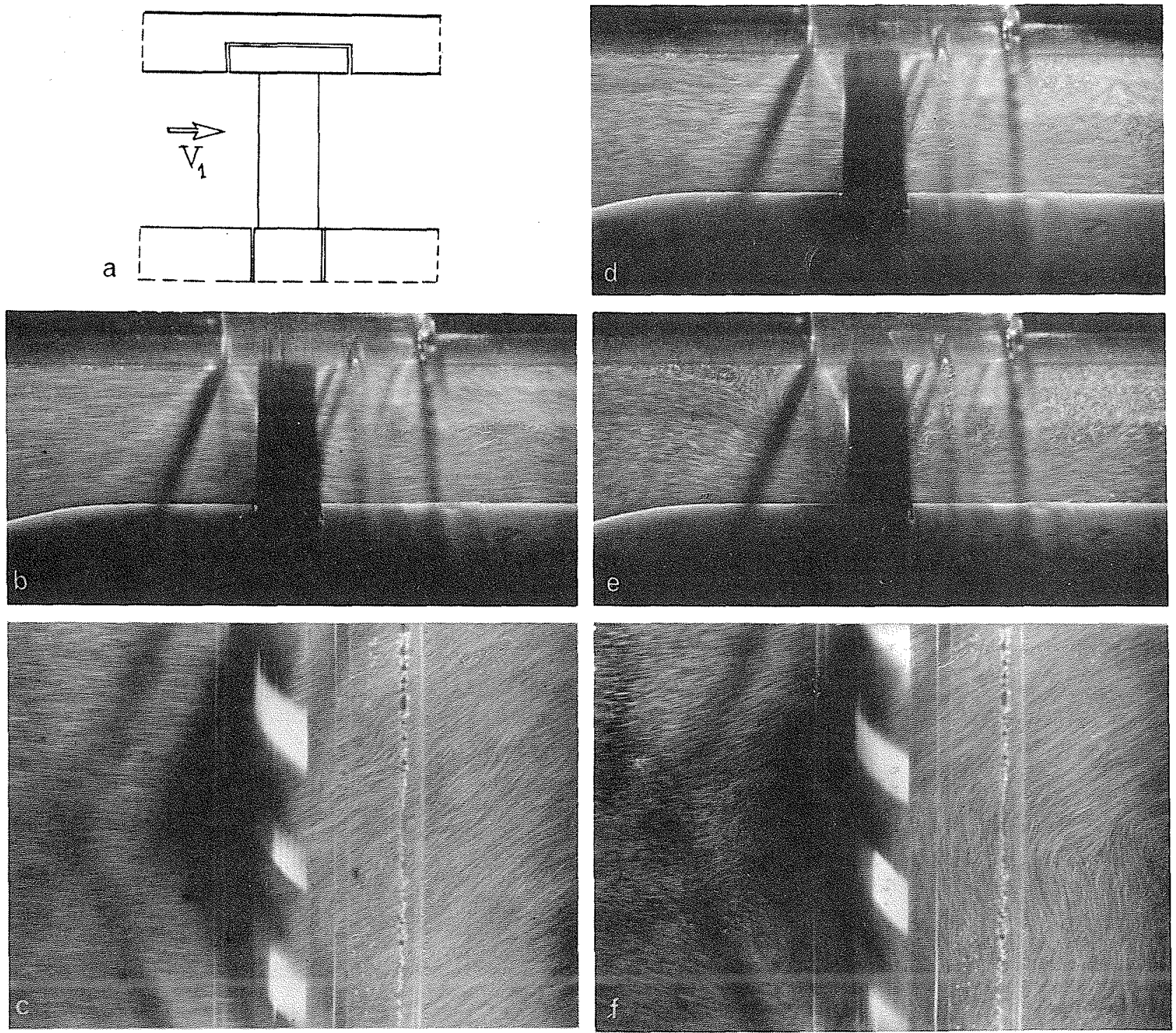


\section{Références - cinémathèque}

[1] FABRI J. et SURUGUE J. - Fonctionnement instable et décollement toumant dans les compresseurs axiaux. Fluid Machinery and Fluidies J.S.M.E., Tokyo, 1972, p. 151-159, voir [11].

[2] PAULON J. - Instabilités d'écoulement dans les compresseurs supersoniques en régime de faible taux de compression - Revue Fr. de Méc. $n^{\circ}$ spécial, 1976, p. 177-184, voir [12].

[3] LARGUIER R. et de SIEVERS A. - Mesures instationnaires dans les turbomachines - Rech. Aérosp., 1975-5, p. 267-277, voir [13].

[4] WERLE H. et GALLON M. - Adaptation de la cuve hydraulique à la visualisation de l'écoulement dans les tur bomachines. Rech. Aérosp., n 1972-1, p. 15-21.

[5] WERLE H. et GALLON M. - Ecoulement dans une maquette hydraulique de turbomachine axiale - Rech Aérosp., no 1977-5, p. 267-288, voir [10].

[6] CHINCHOLLE L. - Visualisation des écoulements relatifs dans les machines tournantes. Rotoscope - La Houille Blanche, $n^{\circ} 1,1968$.
[7] WERLE H. - Le tunnel hydrodynamique au service de la recherche aérospatiale - Publication ONERA $n^{\circ} 156$, 1974.

[8] WERLE $H_{0}$ et GALLON M. - Sillages de cheminées, faisceaux tubulaires, grilles et turbomachines. Quelques exemples de visualisations basées sur l'analogie hydraulique - La Houille Blanche $n^{\circ} 4,1973$.

[9] WERLE H. - Transition et décollement : visualisation au tunnel hydrodynamique de l'ONERA - La Rech. Aérosp., 1980-5, p. 331-345, voir [14].

[10] Film ONERA $n^{\circ} 707$ (1981) - Ecoulement dans une maquette de turbomachine axiale (15 $\mathrm{mn})$.

[11] Film ONERA no 722 (1975) - Analyse des écoulements dans les turbo-compresseurs ( $25 \mathrm{mn}$ ).

[12] Film ONERA $\mathrm{n}^{\circ} 834(1976)-$ Ecoulements instation naires dans les turbomachines $(20 \mathrm{mn})$

[13] Film ONERA $n^{\circ} 823$ (1981) - Visualisations et sondages dans des grilles d'aubes mobiles $(5 \mathrm{mn})$.

[14] Film ONERA $n^{\circ} 930$ (1978) - Couches limites (une étude phénoménologique à l'aide de visualisations au tunnel hydrodynamique) ( $32 \mathrm{mn})$.

Nota : tous ces films (16 mm, sonores français et anglais) figurent sur catalogue édité par l'ONERA : s'adresser au service des relations publiques.

\section{Discussion}

Président: J. PAULON

Le Président. - Je remercie M. WERLE pour ce film très beau et très instructif.

Avez-yous des questions à lui poser ?

M. Maurice ROY (Membre de l'Institut). - Je voulais particulièrement assister à cette présentation, parce que j'ai initié puis encouragé, il y a déjà une trentaine d'années la réalisation et les essais du laboratoire où M. WERLE a développé toutes oes études avec un perfectionnement constant et en abordant, maintenant, le problème encore plus difficile d'une machine tournante.

Il y a, dans les photos et dans le film que nous venons de voir, des indications très intéressantes, lesquelles ne correspondent pas du tout aux schématisations intuitives de certaines personnes bien intentionnées, mais qui veulent élaborer prématurément une théorie, c'est-à-dire sans avoir "vu" le phénomène et sans avoir tenté de comprendre son mécanisme, et le schématiser par une représentation mathématique.

Les essais de M. WERLE sont naturellement exécutés avec un nombre de Reynolds très faible par rapport à celui de turboréacteurs d'aviation, ou des grands turbo-alternateurs de l'industrie. Ce n'est donc pas transposable directement aux nombres de Reynolds et aux nombres de Mach qui sont pratiqués en ces machines. Mais ces essais servent d'une manière fondamentale, à mon avis, à éclairer la compréhension du mécanisme des écoulements fluides complexes et même très complexes pour lesquels les observations ordinaires, voire les plus modernes appareils d'enregistrement de mesure, ne donnent que des moyennes.

C'est pourquoi je tiens à lui recommander de persévérer en cette étude où son talent de réalisateur égale son érudition professionnelle.

M. GUITON. - Je voudrais d'abord remercier M. WERLE car ce film a dépassé notre attente. Il faudrait certainement le revoir plusieurs fois pour en tirer tout le profit nécessaire.

$M$. WERLE. - Il est à la cinémathèque de l'ONERA. Il peut être acquis ou emprunté auprès du Service des Relations Publiques.
M. GUITON, - Il me permet de mieux comprendre la corrélation entre ce qu'on a étudié ce matin, la pré-rotation, et les décollements tournants. Il n'y a pas de contradiction. Dans vos dernières figures, on voit que si l'on augmente l'amplitude de phase, l'amplitude en azimut, on arrive à la prérotation sur toute la périphérie.

Enfin, remarque de détail... je suppose que $œ$ qui est sur votre schéma mais qu'on ne voit pas, en haut, c'est un arbre d'entraînement, la machine fonctionnant en compresseur, et on pompe dans le cas particulier; il doit y avoir un petit moteur en haut.

M. WERLE. - Oui. Les deux vitesses sont indépendantes. On règle comme on veut la vitesse débitante et la vitesse de rotation. On peut réaliser tous les cas de figure désirés.

Je tiens à dire quand même que certains essais avec rotoscope n'ont pas été faciles... et je m'étais un peu imprudemment cmbarqué dans ce procédé.

M. ROY. - Oui, c'est de l'acrobatie expérimentale. Mais les belles réalisations d'études expérimentales sont, presque toujours, plus ou moins acrobatiques.

$M$. WERLE. - J'ai été obligé de tenir compte de vos conseils et de réaliser des schémas. Je pense que le montage du rotoscope n'aurait pas été compris sans schéma.

M. ROY. - Les schémas que vous avez présentés en couleur sont nettement améliorés par rapport à ceux que vous présentiez autrefois en noir et blanc. Ils sont vraiment illustratifs. Je pense que ces images font une excellente publicité à la qualité de la recherche française en ce domaine.

Je dois dire qu'il y a plus de vingt ans j'ai eu l'occasion, sur invitation de l'Académie des Sciences de Moscou, de présenter en l'Université de cette ville, un film que j'avais fait réaliser spécialement à l'ONERA, en ce laboratoire hydrodynamique, sur les principaux phénomènes illustratifs de "pertes de charge". Cette présentation connut un grand succès, au point que je fis don de ce film pédagogique à cette Université car il avait été traduit en russe. 
M. CANAVELIS. - Le film de M. WERLE semble faire apparaître, à débit partiel, un décollement situé à la ceinture de la machine tant dans la section d'entrée de la roue que dans la section de sortie comme indiqué sur le schéma $\mathrm{A}$.

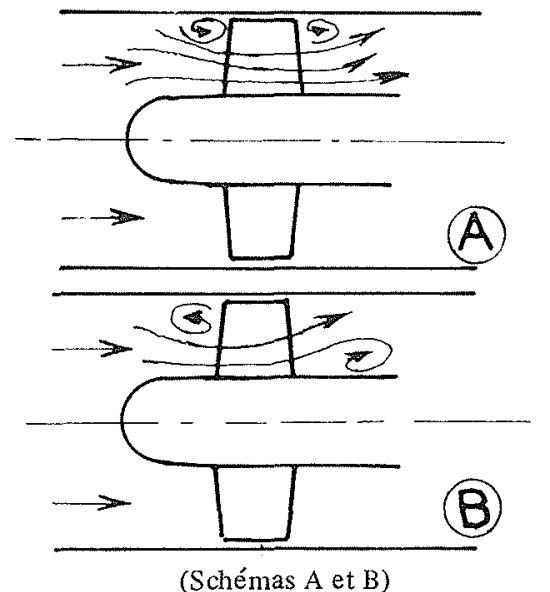

Il convient de noter que dans les pompes axiales, nous avons toujours observé une situation conforme au schéma $\mathrm{B}$, avec, dans la section de sortie de la roue, un décollement vers le moyeu et non pas vers la ceinture. Il serait donc intéressant de mettre en évidence les raisons d'une telle différence de comportement. A ce jour, la question reste ouverte.

M. WERLE. - Dans les conditions des essais réalisés pour ce film, le décollement tournant a toujours été observé côté carène en amont comme en aval.

M. Marc ANDRE. - Je crois que ce film est en effet du point de vue pédagogique une petite merveille; mais il ne traduit pas forcément une réalité, et en ce qui concerne les ventilateurs, les travaux du Professeur JAUMOTTE que j'ai vus à Bruxelles étaient faits avec un capteur piézométrique; il n'y avait pas une visualisation comme cela, mais il pouvait sonder verticalement en amont. Pour un ventilateur, le décollement tournant se formait parfois au pied de pale. Cela dépend de la forme de l'aube. Ici, vous avez affaire à une aube qui n'est pas vrillée, et le décollement s'amorce plutôt en haut qu'en bas. Pour les ventilateurs, le décollement tournant s'amorce en général à la base de la pale.

$\mathrm{Je}$ ne sais pas comment les choses se passent pour une turbine hydraulique, mais pour un ventilateur axial, c'est en général au pied de pale que le phénomène s'amorce.

Le Président. - A l'ONERA, nous avons fait de nombreux essais sur des compresseurs axiaux, et même sur des ventilateurs. Il peut arriver que le décollement prenne naissance en pied, mais dans 90 ou $95 \%$ des cas que nous connaissons il prend naissance en tête. Mais je suis d'accord que la forme des pales influe.

M. le Professeur CHAIX. - Voici une esquisse de l'écoulement méridien visualisé grâce à un éclairage par tranche sem-

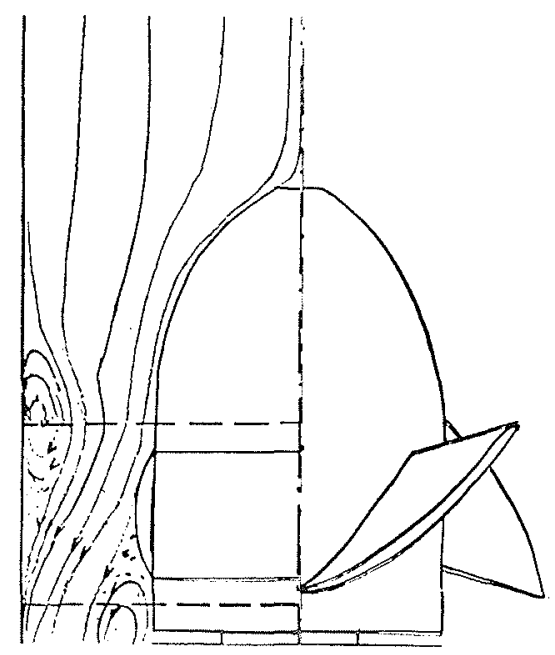

blable à celui de M. WERLE. Seule différence : de la poudre d'aluminium à la place de bulles d'air. Il ne s'agit pas de décollement tournant : les deux tourbillons annulaires disposés selon le schéma $B$ et $M_{0}$ CANAVELIS sont typiques pour un débit réduit de moitié.

La photographie suivante, prise avec la même technique, montre des zones de recirculation comparables, dans un joint à laby rinthe.

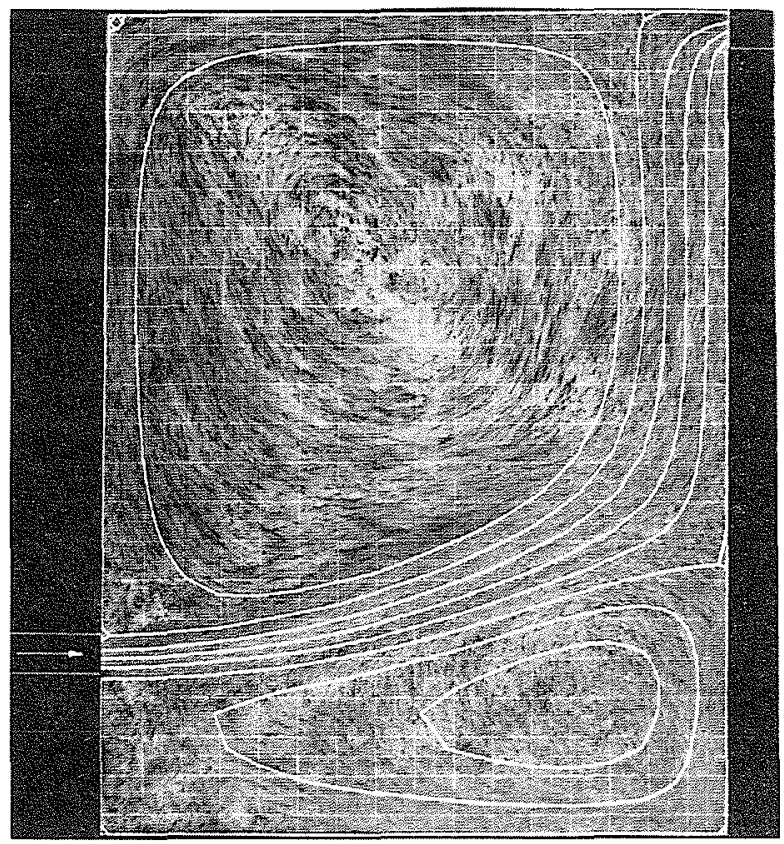

Les lignes de courant superposées ont été calculées avec un programme de Spalding, adapté par l'Ecole Polytechnique Fédérale de Lausanne et par nous-même. Je pense que de tels programmes de calcul pourraient contribuer à l'analyse des phénomènes discutés pendant cette session.

Le film de $M$. WERLE est alors projeté une seconde fois.

M. CANAVELIS. - Cette fois on a très nettement vu qu'à l'aval comme à l'amont le décollement était bien côté carène... contrairement à ce qu'on a l'habitude de voir ou de croire, s'agissant des pompes.

Ici, il s'agit bien d'eau, mais avec un nombre de Reynolds peut-être un peu faible.

$M$. WERLE. - Il y a peut être une épaisseur de la couche limite côté carène et côté moyeu qui intervient.

M. SCHIAVELLO. - C'est le rapport entre la corde et la hauteur de l'aube qui importe.

M. WERLE. - Le rayon du moyeu est de $100 \mathrm{~mm}$; le rayon du carter est de $146 \mathrm{~mm}$. Donc la hauteur de l'aube est, compte tenu du jeu, de l'ordre de $45 \mathrm{~mm}$ et la corde fait $50 \mathrm{~mm}$.

M. SCHYAVELLO. - Il faut faire attention parce qu'il y a des pompes axiales où l'envergure des aubes est alors beaucoup plus grande que la corde. Dans ces conditions, on peut avoir un tourbillon tournant à l'entrée, à la ceinture, et un tourbillon tournant à la base de l'aube, à la sortie. C'est le paramètre "hauteur de l'aube sur la corde" qui est le paramètre déterminant pour la configuration des tourbillons à l'entrée et à la sortie.

Le Président. - Je remercie vivement les présentateurs de cette session et les nombreux participants aux discussions. La Séance est levée. 
Abstract

Visual hydrodynamic study of flow in a scalemodel representing an axial turbomachine

To enhance the knowledge of the penomena characterising flow in an axial turbomachine, visual inspection in a hydrodynamic tunnel was performed by the ONERA, drawing the existing analogy between aerodynamic and hydraulic flow conditions. The visual scrutiny of walls and space is obviously easier and finer in water than in air, especially in non-stationary state.

The scale-model used is vertical and includes a mobile runner fitted with 20 cylindrical vanes (circumference $1=50 \mathrm{~mm}$ ) placed in a cylindrical channel (radius of casing $R=146 \mathrm{~mm}$ ).

As the flow velocity $V_{1}$ and the speed of rotation $N$ of the runner are controlled and regulated independently, all operating conditions can be simulated using this assembly. In addition to the main testing layout (isolated mobile runner), a few variations have also been tested (mobile runner with a rectifier, mobile runner with a rotating keel).

The assembly has been designed to the used for the following scanning methods:

- emissions of a coloring agent of density 1 produced from fixed or mobile elements (carcen, hub, vanes, etc.)

- air bubles in suspension in the water. They are observed through lit sections placed logitudinally (diametrically cut or half-way up the vanes) or transversally (cut at the edge of leakage of mobile vanes or half-way along the chord of the rectifier blades).
The power and efficiency of the stage are a function of the volume flow and the percentage losses by comparison with optimum power due to ventilation.

The transition zone between motor and receiver modes of the stage is approximately $(Q V)^{*}=0.5$.

Maximum power intake is a major share of optimum power, especially when the variation of $(Q V)^{*}$ is associated with an increase in downstream pressure.

The hypotheses adopted and the analytical findings are in agreement with experience and yield an acceptable accurate forecast of the performance of a turbine stage under partial load.

The conclusion from the various observations made is that terminal stage flow is highly sensitive to the load variations necessitated by its environment.

A separated flow rotating en bloc with the mobile blading occurs at the downstream section of the blade when the volume flow is reduced to $25 \%$ below its rated value. The stall then increases and forces the flow to the central part and the periphery up to the point at which a second toric vortex is formed upstream and at the tip of the blade. This eddy rotates at virtually the same speed as the mobile blading.

At zero flow, the two eddies rotating in opposite directions invade the channel and produce losses called "losses due to ventlation", in which the centrigual effect is dominant. 